\title{
The geomagnetic semiannual anomaly on the four Dst-fundamental observatories: Dependences with Sun-Earth physical parameters
}

\author{
Francisco Azpilicueta, ${ }^{1}$ Claudio Brunini, ${ }^{1}$ and Emilio Camilión ${ }^{1}$ \\ Received 15 March 2012; revised 23 May 2012; accepted 24 May 2012; published 6 July 2012.
}

[1] The semiannual anomaly (also known as semiannual variation) on the magnetic activity is a phenomenon that produces clear minima during March and September and maxima in June and December on the horizontal components of the geomagnetic field. This phenomenon has been known since the middle of the nineteenth century, but in spite of the accumulation of measurements and the development of three theoretical models, a conclusive physical explanation for it has not been developed. The usual approach to study the semiannual anomaly is by means of geomagnetic indices like the disturbance storm time, Dst, which is based on combining measurements registered on four magnetic observatories. This work follows a different approach based on the raw horizontal components registered at the four observatories. The analyses performed aimed to study and assess the impact of several external parameters, characteristics of the Sun-Earth environment, on the semiannual anomaly. The influence of the global geomagnetic activity level, the solar activity level, the solar magnetic polarity, and the rising/declining phase of the solar radiation cycle is analyzed in detail. The most important finding is that the semiannual anomaly is always present and that none of the previously mentioned parameters significantly favor the development of it. A second result is the presence of a 27 day signal superposed to the semiannual anomaly which is significantly affected by the solar activity level.

Citation: Azpilicueta, F., C. Brunini, and E. Camilión (2012), The geomagnetic semiannual anomaly on the four Dst-fundamental observatories: Dependences with Sun-Earth physical parameters, J. Geophys. Res., 117, A07204, doi:10.1029/2012JA017730.

\section{Introduction}

[2] The semiannual anomaly on the magnetic activity is a phenomenon known for a long time. One of the first reports about it dated back to the middle of the nineteenth century, to the pioneering work of Sabine [1856]. The manifestation of this phenomenon is a clear semiannual variation in the magnetic field measured on the terrestrial surface with deep minimums in March and September (close to the equinoxes) and maximums in June and December (close to solstices). The expression semiannual anomaly corresponds to the aeronomic community, while within the community of geomagnetic physics the same phenomenon is known as the semiannual variation.

[3] Since Sabine's report, science and technology have taken several important steps forward. During this time researchers have systematically observed the geomagnetic semiannual anomaly and spent effort to understand the

${ }^{1}$ CONICET, Facultad de Ciencias Astronómicas y Geofísicas, Universidad Nacional de La Plata, La Plata, Argentina.

Corresponding author: F. Azpilicueta, CONICET, Facultad de Ciencias Astronómicas y Geofísicas, Universidad Nacional de La Plata, Paseo del Bosque S/N, La Plata, Buenos Aires B1900FWA, Argentina. (azpi@fcaglp.unlp.edu.ar)

C2012. American Geophysical Union. All Rights Reserved. 0148-0227/12/2012JA017730 physical mechanisms that is behind it. The historical benchmarks associated to the development of new theories on this anomaly are (i) the "axial hypothesis" proposed by Cortie [1912], which has the heliographic latitude of the Earth as the driven parameter, (ii) the "equinoctial hypothesis" that was first proposed by Bartels [1932], in which the driven parameter is the tilt angle between the Sun-Earth direction and the geomagnetic dipole, and (iii) the "Russell and McPherron hypothesis" presented in Russell and McPherron [1973], in which the main parameter is the southward component of the interplanetary magnetic field.

[4] During the last years several authors [e.g., Lal, 1998; Lyatsky and Tan, 2003; O'Brien and McPherron, 2002; Cliver et al., 2002] have dedicated some effort to assess which theory dominates in the process and presently there is a tendency to accept that the "equinoctial hypothesis" plays the most important role on generating the anomaly, although the associated physical mechanism has not yet been identified. Other authors [e.g., Clúa de Gonzalez et al., 2001] have studied the possibility of simultaneous occurrences of the three known mechanisms. According to Häkkinen et al. [2003] the Russell-McPherron effect seems to be the dominant one and that the combined contributions of the three mechanisms can only explain $50 \%$ of the observed variability. The three hypotheses agree on that the anomaly is caused by interactions between solar wind charged particles, 


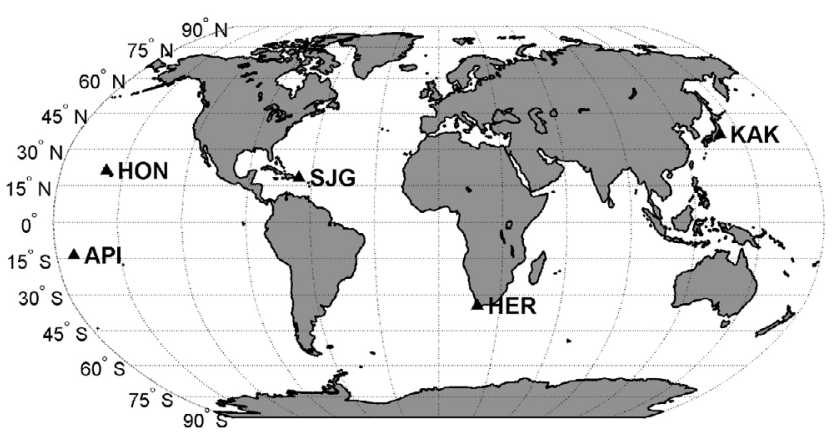

Figure 1. Geographical distribution of the four Dstfundamental observatories (HON, SJG, KAK, and HER) and the additional observatory, API, used in this work.

the geomagnetic field and the ring currents in the surrounding environment of the Earth. In spite of the several decades since its discovery and the theoretical developments, there is no definitive explanation for the occurrence of this phenomenon.

[5] Most of the works found in the specialized literature about the anomaly are based on the study of geomagnetic indices, which are computed by averaging one or a combination of the magnetic field components measured at a set of magnetic observatories [Yoshida, 2009; Cliver et al., 2002, 2004; Lal, 1998; Chaman-Lal, 2000; Russell and McPherron, 1973; Russell, 1989; Svalgaard, 1977, 2011]. Among these indices, it can be mentioned the $a a, \mathrm{~K}, \mathrm{Kp}, \mathrm{U}$, $D s t$, etc. For a complete and detailed description about geomagnetic indices [see Mayaud, 1980]. One of the most widely used indices for studying the semiannual anomaly is the Dst, because it is comparative simple to compute and interpret. Just to mention a representative sample of works based on Dst, we can be mentioned [Cliver et al., 2001; Clúa de Gonzalez et al., 2001; Häkkinen et al., 2003; Lyatsky and Tan, 2003]. A further description of the Dst is included in section 2. The number of works dealing with the semiannual anomaly from direct raw measurements of the magnetic field is low compared to the number of papers based on geomagnetic indexes. This contribution presents a study of the semiannual anomaly based on the horizontal component of the magnetic field measured at the four Dst-fundamental magnetic observatories.

[6] The first goal of this work is to better understand the individual contribution of each observatory to the formation of the semiannual anomaly on the Dst. For doing this, a technique was developed to reconstruct the 'annual pattern' of the horizontal component ( $\mathrm{H}$ component) observed at each observatory. The dominant feature of this reconstructed pattern is the semiannual anomaly. Section 3 starts with a detailed description of the technique and ends with a discussion about the results found. The most noticeable element coming from this analysis is a significant difference in the annual pattern of the Southern observatory, HER.

[7] Section 4 presents evidences that the difference observed in HER observatory is not associated to any peculiarity of the observatory location, but it is intrinsic of the Southern Hemisphere. Processing the data series of a fifth observatory (located in the Southern Hemisphere), we obtained an annual pattern with the same characteristics of HER. The results confirm the existence of an interhemispheric asymmetry.

[8] The second goal of this work was to study and assess the impact of several external parameters, characteristic of the Sun-Earth environment, on the annual patterns. Section 5 contains a thorough description of the analysis performed to evaluate the influence of the general geomagnetic activity level, the solar activity level, the solar magnetic polarity and the phase (rising or declining) of the solar radiation cycle.

[9] Finally, section 6 summarizes the most important findings about the impact of the external parameters and makes a general conclusion and implications about the results.

\section{Dst Index and the Fundamental Magnetic Observatories}

[10] The disturbance storm time (Dst) index is one of the most important solar-terrestrial indices. It measures the hourly variations of the magnetic field at the surface of the Earth and has been continuously computed at the World Data Center WDC-C2 at Kyoto, Japan, since the International Geophysical Year 1957. This index is computed basically by averaging the deviation from reference values of the $\mathrm{H}$ component at four fundamental geomagnetic observatories located at low to middle latitudes: Hermanus (HER), South Africa, Honolulu (HON), Hawaii, Kakioka (KAK), Japan, and San Juan (SJG), Puerto Rico. For details on the Dst computation the interest reader is referred to [Sugiura and Kamei, 1991; Häkkinen et al., 2003]. Figure 1 shows the geographical distribution of the geomagnetic observatories contributing to the Dst computation. Although the Dst is commonly used to detect and study geomagnetic storms, it is also widely used as a measure of the intensity of the ring current that surrounds the Earth at distances between 1 to 3 earth radii in the equatorial plane. The $D s t$ index values used in this work are publicly available at http://wdc.kugi. kyoto-u.ac.jp/dstdir/.

\section{Reconstruction of the Semiannual Pattern}

[11] This section presents the processing scheme applied to the long-term data series of each of the standard geomagnetic observatories. Figure 2 shows the daily mean values of the $\mathrm{H}$ component of the magnetic field for each observatory. For clarity, the value for 1 January 2000 for each series was chosen as reference, $\mathrm{H} 0$ (10719.58 $\mathrm{nT}$ for HER, $27572.92 \mathrm{nT}$ for HON, $27057.96 \mathrm{nT}$ for SJG and 30002.29 $\mathrm{nT}$ for KAK).

[12] Two main features can be mentioned from this figure: (i) the $\mathrm{H}$ component shows completely different behaviors depending on the observatory location and on the year and (ii) the series from HON and SJG shows jumps that cannot be attributed to natural processes; instead, they must be the results of changes in the configuration of the magnetometers and/or on the calculation of the baseline for the observatory. As will be presented in the next paragraphs the technique used in this work is based on annual variations so the jumps do not constitute a problem.

[13] The objective of this work was to analyze the semiannual anomaly on the magnetic activity, so a technique for removing the trend was applied that consisted on modeling each year independently with a linear function of the day of 

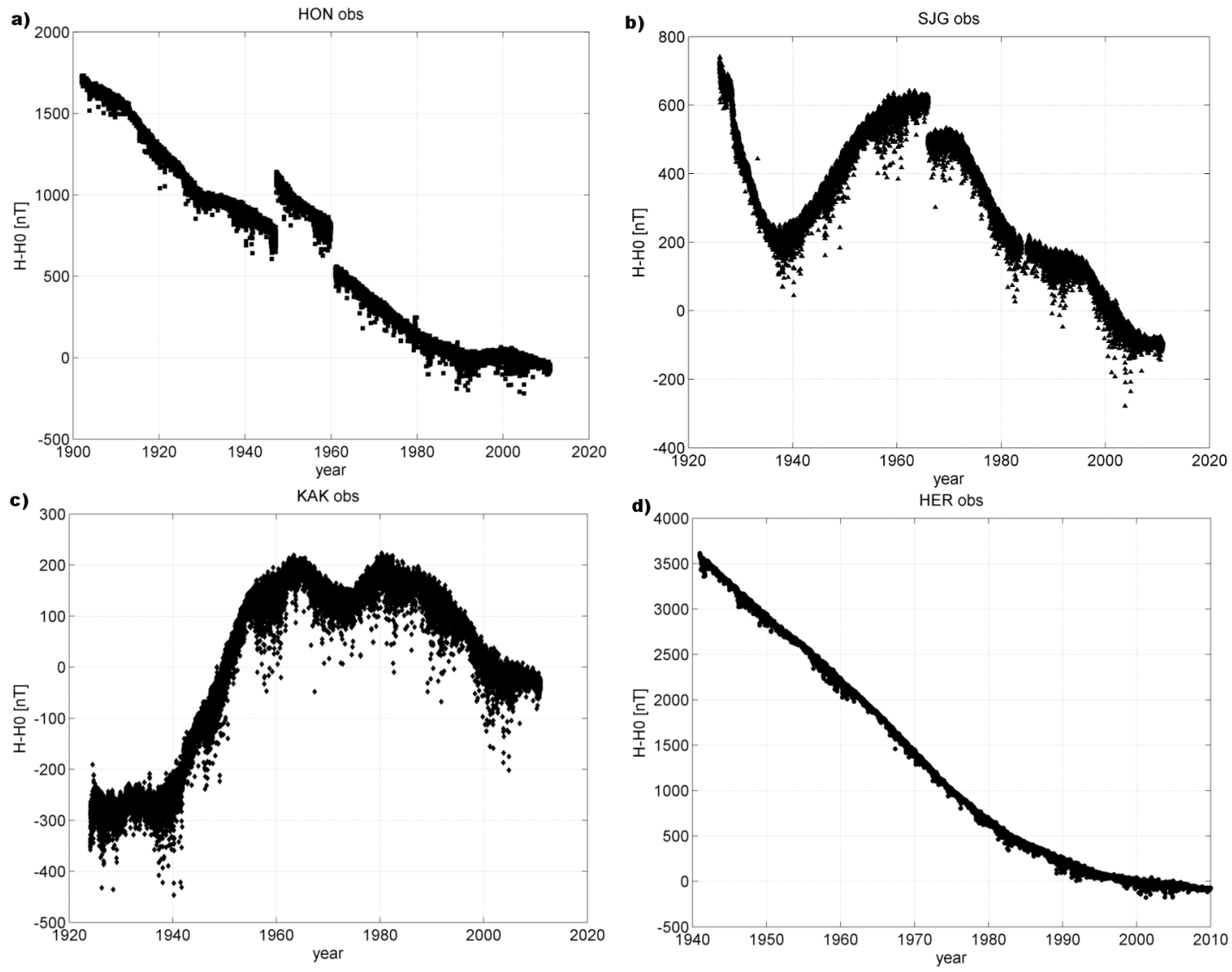

Figure 2. Daily means of the H-H0 of the four standard magnetic observatories: (a) HON, (b) SJG, (c) KAK, and (d) HER. The values for H0 are 27572.92 nT for HON, $27057.96 \mathrm{nT}$ for SJG, $30002.29 \mathrm{nT}$ for KAK, and10719.58 nT for HER.

year (DOY). This method is equivalent to modeling the trend with a piecewise linear function and is quite different from the one usually applied consisting on fitting a quadratic function to a 5 year data set, e.g., the procedure used in computing the Dst index [Sugiura and Kamei, 1991]. We found that the year basis method is more suitable for our study because it does not introduce any artificial bias coming from the difficulty to model the general trend with a polynomial function that only depends on the year. After subtracting the trend from the daily mean values a deviation of the $\mathrm{H}$ component from the expected value given by the modeled trend is obtained. In other words the original data series has been low band pass filtered, leaving only those signals with periods equal to or lower than a year. Figures $3 a-3 d$ show the deviations for the four observatories as a function of year. For clarity purposes, a moving average with a 30 day window was applied. For comparison purpose Figure 3e shows the temporal series of the F10.7 index. This index, which is publicly available at ftp://ftp. ngdc.noaa.gov, gives a measure of the solar radiation activity level and will be described with details in section 5.2.

[14] The first interesting result that comes out from Figure 3 is the clear signature of the solar cycles (widely known as the 11 year solar cycle). The effect of this cycle is a modulation of signal with periods less than 1 year leading to periods of maximum amplitude approximately every 11 years synchronized with the solar activity. For example consider the last solar peak that occurred between years 2001 and 2002. It is also important to note that the measured magnetic field is very sensitive to the level of the solar activity. A clear example is the much diminished peak registered by the four observatories during the peak of solar cycle 20 (occurred by the end of 1968), which was also registered as the lowest peak in the F10.7 index. This issue will be developed with details in section 5.2.

[15] When the deviations of a particular observatory are represented in a composite annual graphic, pictures like the one depicted in Figure 4 are obtained. This figure shows only the deviations obtained for HER observatory mapped against DOY but the graphics obtained for the other three observatories are quite similar. The main feature that immediately appears is a thick curved line with two minimums at DOYs 90 and 290 approximately and two maximums at DOYs 190 and 365.

[16] Then, calculating a mean deviation for each DOY for each observatory we obtained the four curves presented in Figure 5. Figure 6 shows the result of combining the four annual patterns in a unique graph for comparison reasons.

[17] From the analysis of these figures several interesting elements of discussion appear: 
a)

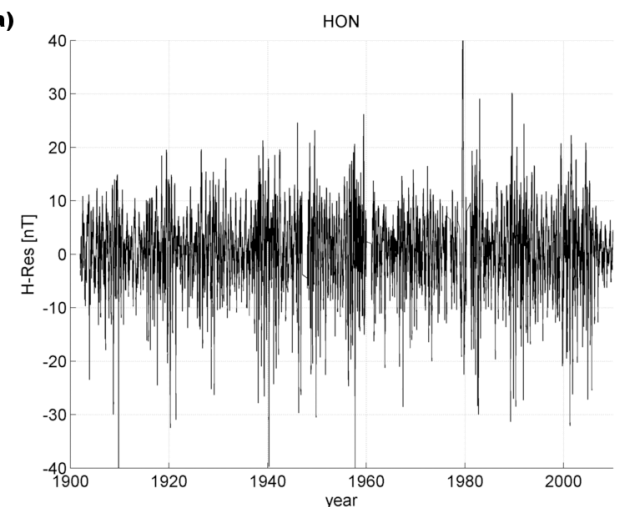

c)

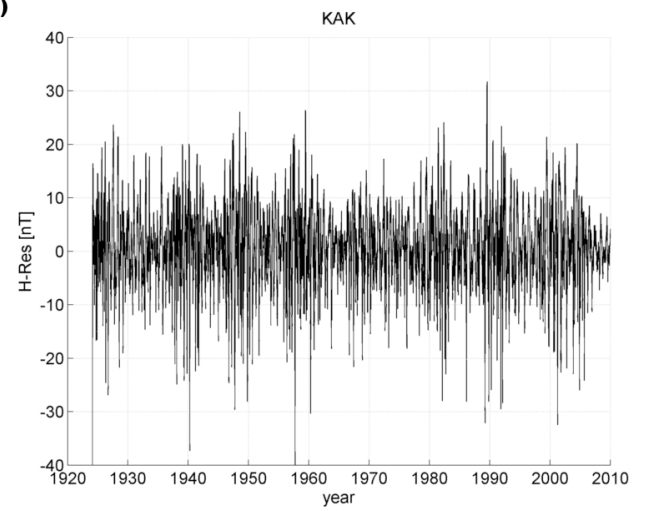

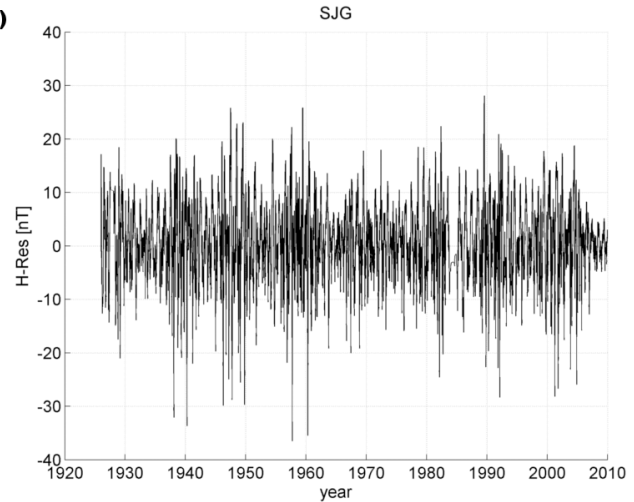

d)

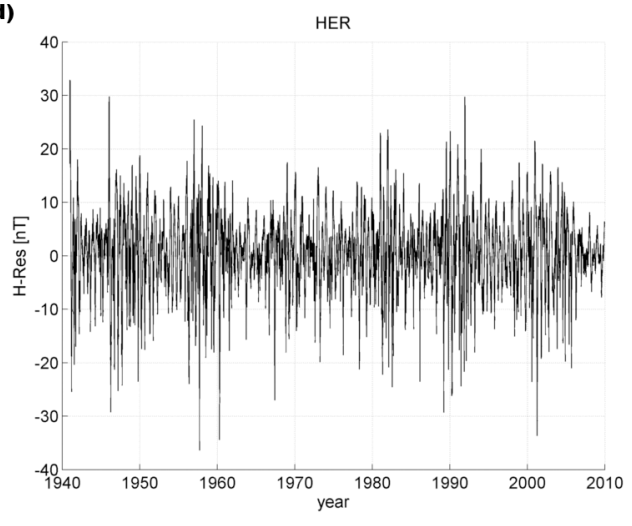

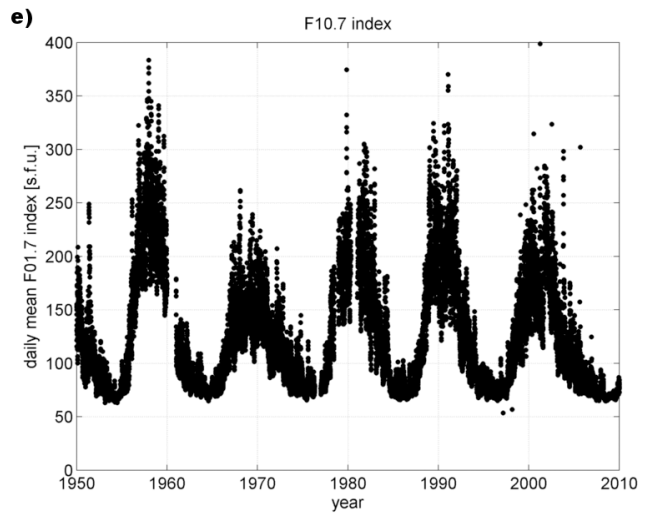

Figure 3. Deviations of the $\mathrm{H}$ component for the four standard magnetic observatories after removing the trend: (a) HER, (b) HON, (c) SJG, and (d) KAK. For clarity purposes a moving average with a 30 day window was applied. (e) Temporal series of the F10.7.

[18] 1. The four observatories share a common annual pattern with minimums at DOYs 90 and 290 approximately (very close to March and September equinoxes) and two maximums at DOYs 190 and 365 (very close to June and December solstices). This constitutes an important confirmation that the semiannual anomaly is measured on the $\mathrm{H}$ component at every site in the middle latitude region, with maximums and minimums occurring simultaneously.

[19] 2. The June maximum is greater than the December one for the three observatories located at the Northern Hemisphere while the December maximum is greater than the June one over HER observatory (Southern Hemisphere). Whether this effect and the semiannual anomaly are associated to the same phenomenon cannot be deduced from these data. Section 4 presents an analysis of this issue.
[20] 3. The correspondence between the patterns of the three Northern observatories is remarkable.

[21] 4. A very distinguishable pattern (a sort of shorter period) occurs between DOYs 50 and 120 that is registered by the four observatories and something very similar occurs between DOYs 270 and 300.

[22] 5. SJG registers a rather flat slope in the first part of the year (up to DOYs 60) when compared with the HON and KAK patterns. This could indicate a second-order dependence of the semiannual on the coordinates of the station, more precisely on the longitude since the latitudes of the observatories are quite similar.

[23] 6. An additional signal with a period of 27 days appears in the pattern of the four observatories. The origin of 


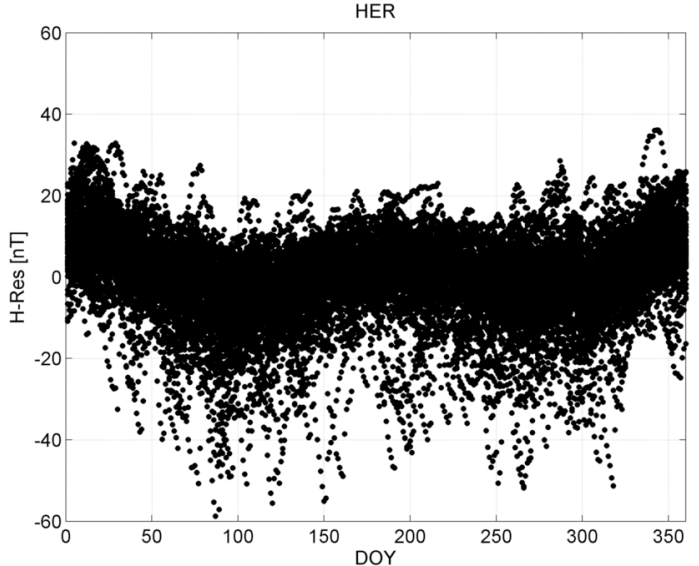

Figure 4. Deviations of the H component for HER observatory mapped against DOY. To reduce the dispersion a 5 day window moving average procedure was applied.

this signal could be associated to the solar rotation. The point will appear clearer at the end of section 5.2.

\section{Interhemispheric Asymmetry}

[24] In the previous section it was observed that the annual pattern of HER observatory presents a significant difference: the December maximum is higher that the June one, in opposition to what is observed on the other three observatories.

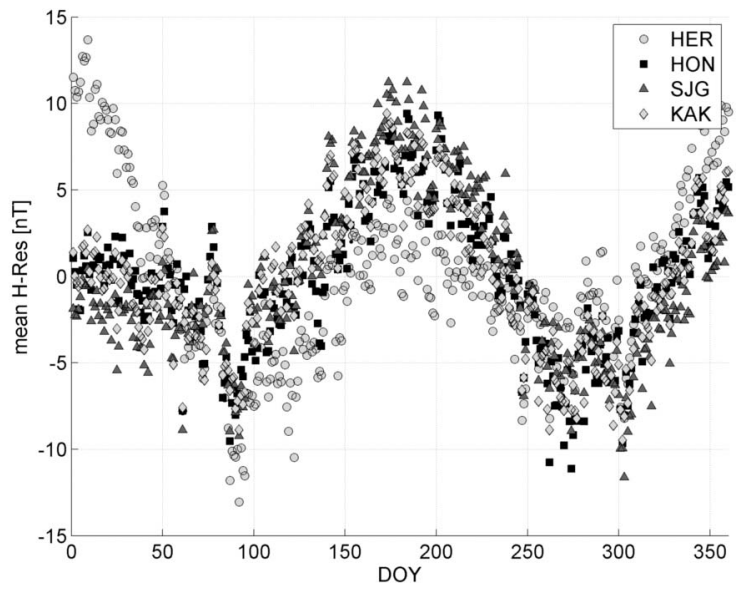

Figure 6. Composite graph of the annual pattern for the four fundamental observatories.

HER observatory is the only of the three that is located on the Southern Hemisphere and this was the main hypothesis to explain the particular observed behavior. In order to verify this hypothesis, the data series of an additional magnetic observatories API (latitude $-13^{\circ} .82$ and longitude $188^{\circ} .21 \mathrm{E}$ ) was processed. API has a long data series that starts in 1921 and extends continuously until the present, is located at the Southern Hemisphere, and corresponds to different longitudinal geographic sectors. a)

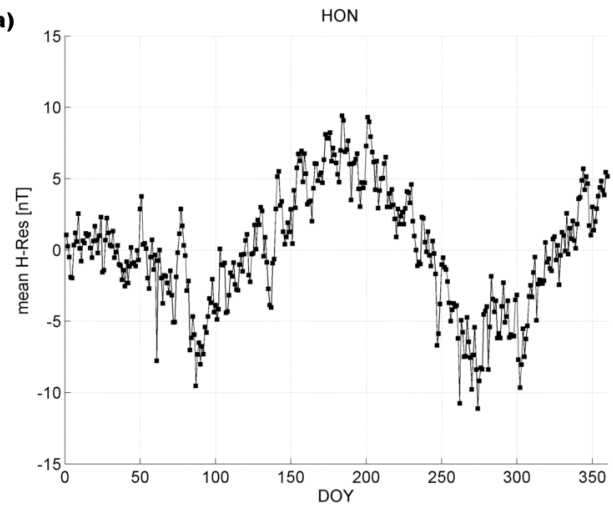

c)

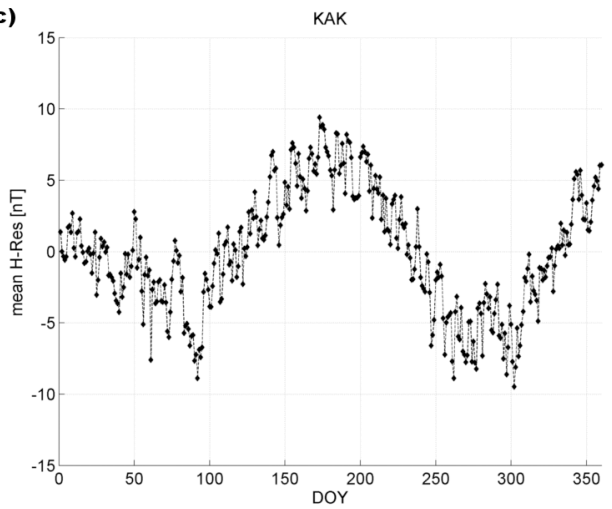

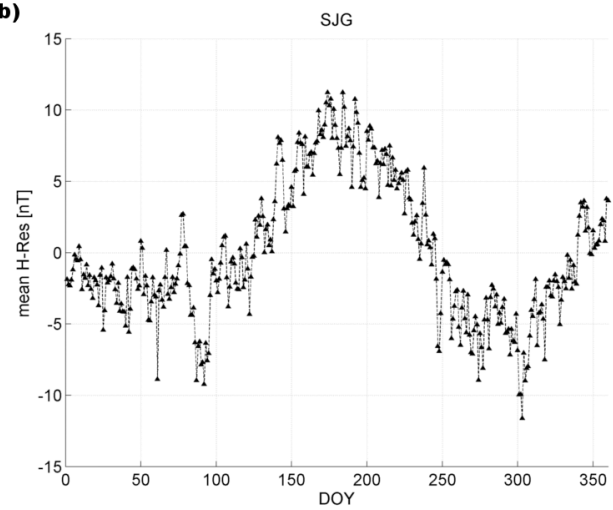

d)

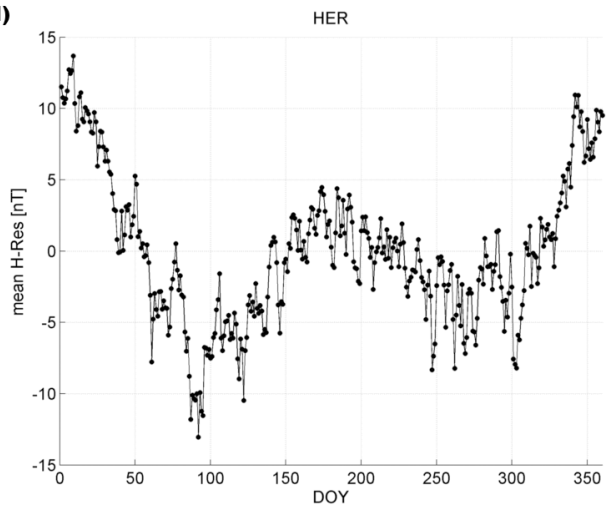

Figure 5. Mean deviations of the daily means of the $\mathrm{H}$ component mapped against DOY for the four fundamental observatories: (a) HON, (b) SJG, (c) KAK, and (d) HER. 


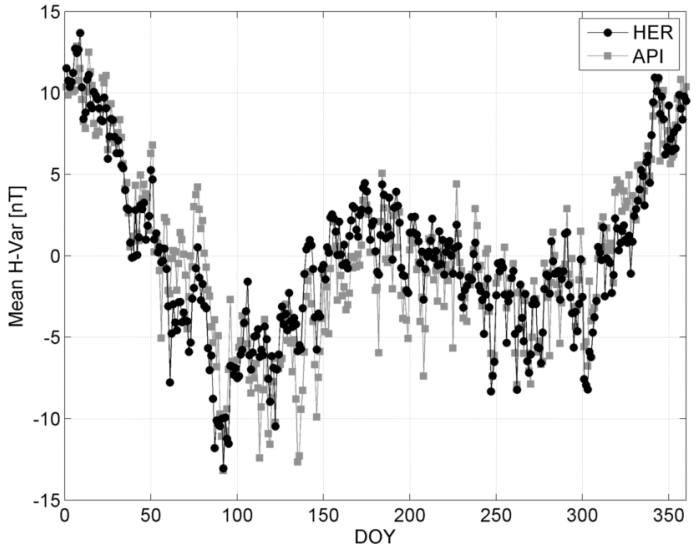

Figure 7. Mean deviations of the $\mathrm{H}$ component for three magnetic observatories located in the Southern Hemisphere. The black dots correspond to HER and gray squares correspond to API.

[25] Figure 7 shows the annual patterns for HER and API. Comparing both curves the main features that come out are (i) the semiannual anomaly with minimums close to the equinoxes is present in both curves and (ii) the condition of the December maximum higher than the June one is verified also on API.

[26] The remarkable similarities of the HER and API annual patterns confirms the hypothesis of significant differences between the semiannual anomaly (or the general annual pattern) observed by Northern or Southern observatories in agreement with the work of Häkkinen et al. [2003]. This element has implications on the interpretation of the Dst index, since in a general sense the Dst index is an average of the data from four observatories, three in the Northern and one in the Southern Hemisphere. Then the behavior of the Dst index will resemble more the behavior of the Northern than the Southern observatories. In other words, the averaging scheme used to compute the Dst might not be enough to cancel out the hemispheric-dependent effects. Häkkinen et al. [2003] proposed the $D s t_{6}$ index, using data from three observatories located on each Hemisphere to balance the effects. A deepen analysis about this issue is currently under work and it would be the subject a future paper.

\section{Dependence of Semiannual Anomaly on Different Sun-Earth Environmental Physical Parameters}

[27] As a general remark of this section the results presented corresponds only to HON and HER observatories. However, HON results must be taken as representative of the three observatories located in the Northern Hemisphere and HER results representative of the Southern observatories.

\subsection{Semiannual Anomaly Dependence on the Geomagnetic Activity Level}

[28] A point of controversy that is frequent matter of discussion is the quantification of the storm and nonstorm contribution to the geomagnetic semiannual anomaly. Several authors [Russell and McPherron, 1973; Mursula et al., 2008] support the hypothesis that the most frequent occurrences of geomagnetic storms during March-April and SeptemberOctober leads to an amplification of the Dst index during these periods of the year causing in this way the minimums of the semiannual anomaly observed in the Dst. This effect is known as the storm contribution. Other authors in opposition [Cliver et al., 2001, 2004] have shown that, although it exists, the storm contribution is not significant and the semiannual anomaly is mainly driven by an effect not related to geomagnetic storms which is called the nonstorm contribution.

[29] For the purpose of analyzing the storm and nonstorm contributions to the results obtained in this work, the procedure explained in section 3 was repeated excluding those days classified as stormy one. Following the quiet storm classification of Gonzalez et al. [1994] we excluded those days with daily mean $D s t<-50$, i.e., those days that presented moderated and strong disturbance behaviors.

[30] Figure 8 shows the annual patterns using data from the period 1957-2010 for HON and HER observatories. The black dots corresponds to the complete data set, while the gray squares represents the annual pattern obtained with the $D s t>-50$ filtered data set. The number of days excluded
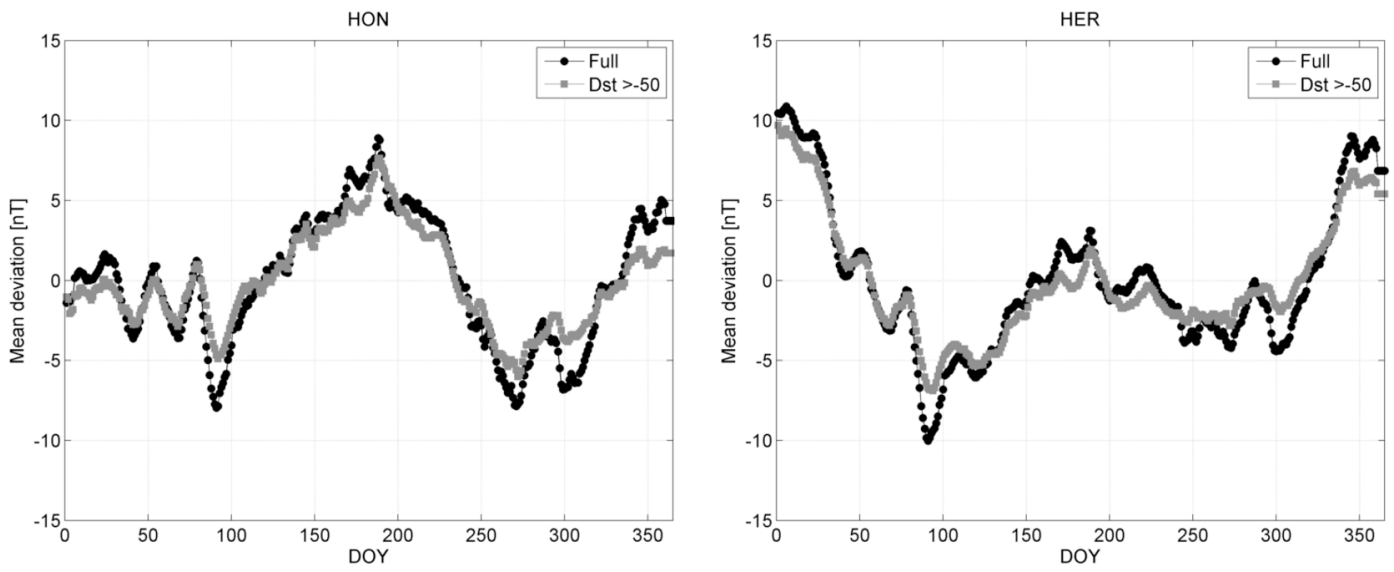

Figure 8. Mean deviations of the $\mathrm{H}$ component for the (left) HON and (right) HER observatories mapped against the DOY. The black dots correspond to the complete data set; the gray squares correspond to the $D s t>-50$ filtered data set. For clarity a moving average with a 10 day window was applied to the data. 

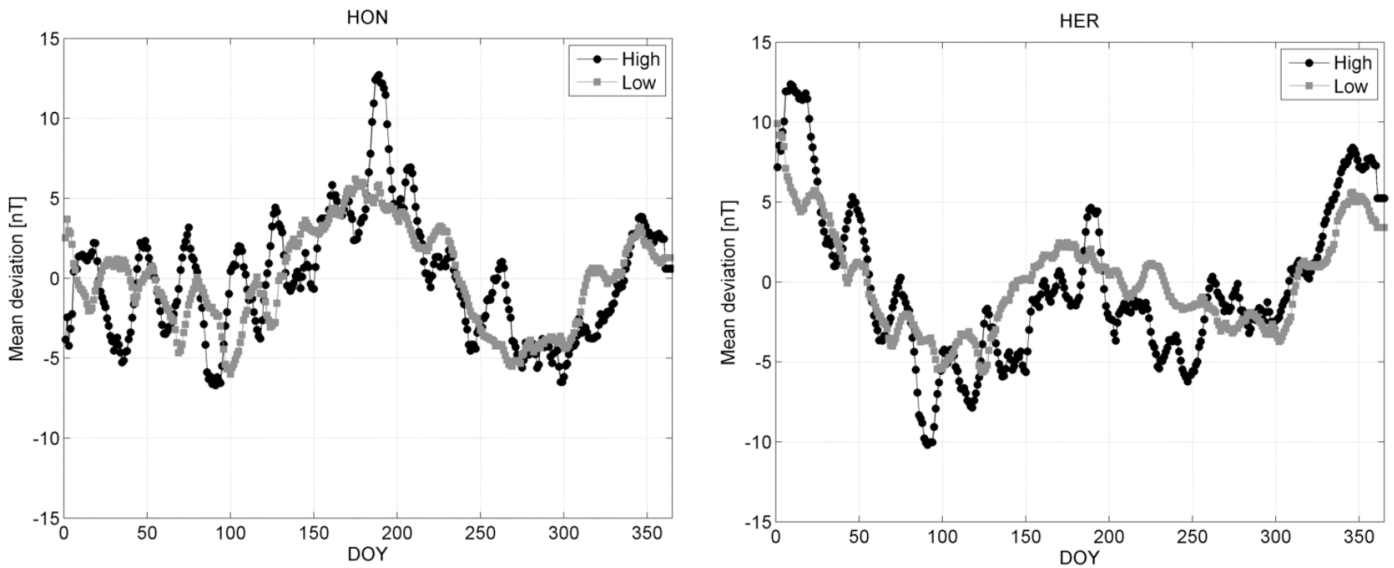

Figure 9. Mean deviations of the H component for (left) HON and (right) HER observatories mapped against the DOY. The black dots correspond to the high solar activity, while the gray squares correspond to low solar activity periods. For clarity a moving average with a 10 day window was applied to the data.

with this criterion was less than $5 \%$. No significant difference between both curves is seen in both observatories (HON and HER). For the full sample there is a slight intensification of the minimums. From this it is quite clear that the nonstorm component is dominant over the storm one. This confirms the idea that the semiannual anomaly is produced by a slowly changing phenomenon with a period of a year (like the proposed by the equinoctial hypothesis mechanism), and it is not related to geomagnetic storms.

\subsection{Semiannual Anomaly Dependence on Solar Activity Level}

[31] Another important physical issue to evaluate is the effect of the solar activity level on the semiannual anomaly. This element has already appeared when discussing the results shown in Figure 3 establishing that the solar activity modulates the amplitude of the high-frequency signals (those with periods less than 1 year).

[32] The F10.7 is an index that measures the intensity of solar radiation at wavelength equal to $10.7 \mathrm{~cm}$. This index is widely used as a proxy of the solar activity level, independently of the phenomena being studied. It is important to clarify that by using this index we are not proposing a direct relationship between the magnetic activity measured at the terrestrial surface and the solar radio emissions at wavelength $10.7 \mathrm{~cm}$. The F10.7 within this work is used as a proxy of the physical phenomena occurring within the Sun that produces the well-known solar cycle (the 11 year cycle).

[33] The followed procedure consisted of binning the data according to three level of solar activity: low activity F10.7 $<90$ s.f.u., medium activity 90 s.f.u. $<$ F10.7 $<120$ s.f.u., and high activity F10.7 $>120$ s.f.u.. A Dst $>-50$ nT filter was also used to exclude the contribution of the stormy days. Figure 9 shows the annual patterns obtained for high and low activity periods for HON and HER observatories. The curves for the moderate solar activity are not shown for clarity reasons but as expected they lay between both depicted curves.

[34] Analyzing Figure 9 it comes out that (i) the semiannual anomaly is clearly seen for any activity level; i.e., it is always present and (ii) the DOYs in which the minimums
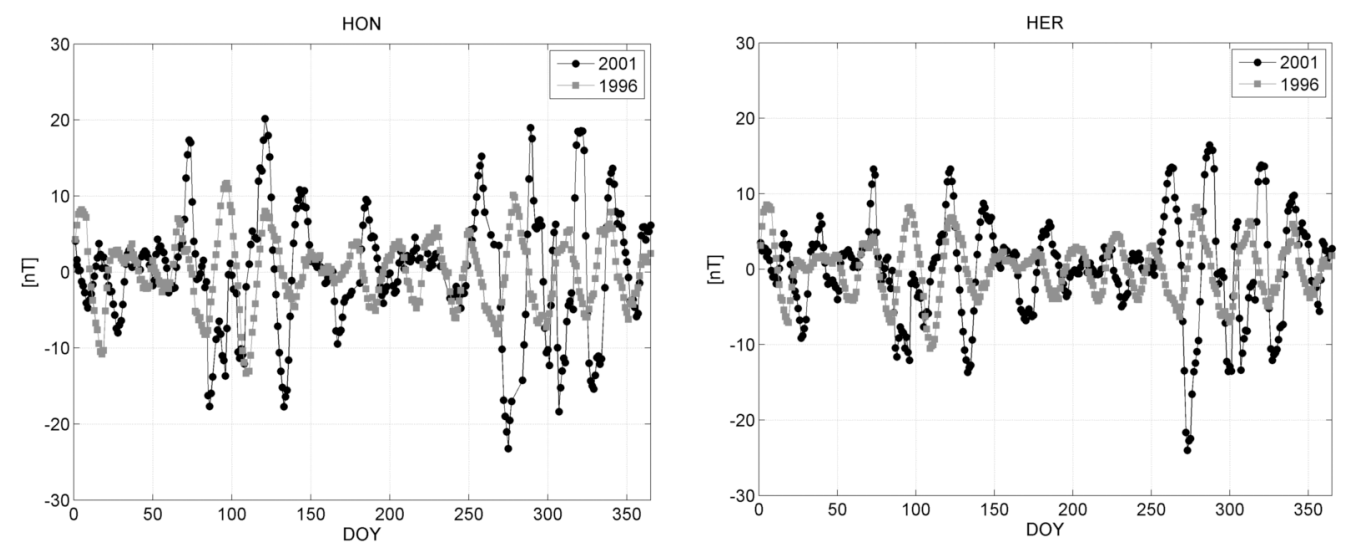

Figure 10. The 27 day signal of the H component for (left) HON and (right) HER observatories mapped against the DOY. The black dots correspond to 2001 (the high solar activity), while the gray squares correspond to 1996 (low solar activity). For clarity a moving average with a 10 day window was applied to the data. 

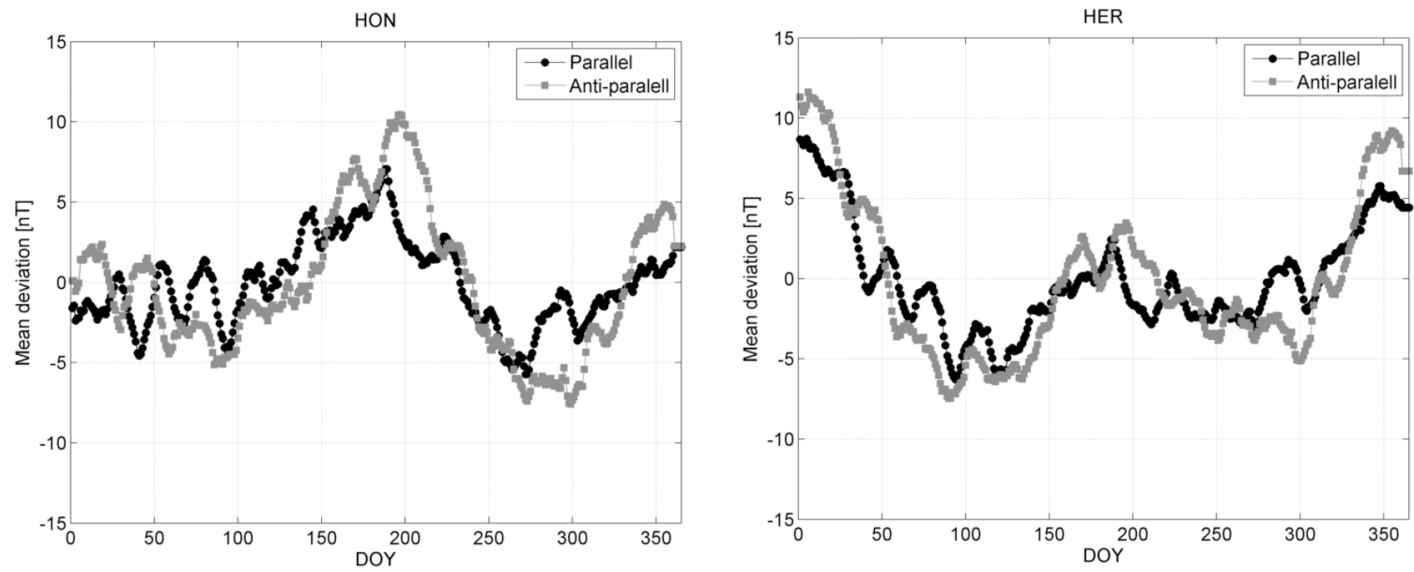

Figure 11. Mean deviations of the daily means of the $\mathrm{H}$ component for (left) HON and (right) HER observatories mapped against the DOY. The black dots correspond to periods of parallel solar magnetic field while the gray squares correspond to antiparallel periods. For clarity a moving average with a 10 day window was applied to the data.

(maximums) occur are the same for both activity periods. But the most important result from this figure is that the solar activity level does not significantly modify the range of variation (minimum to maximum) of the semiannual anomaly.

[35] A closer look of Figure 9 reveals a rather unexpected result: there is a clear effect of the solar activity level on the 27 day signal. To further analyze this, Figure 10 shows the 27 day signal (with 13 peaks per year) obtained for HON and HER for 1996 and 2001, after a low- and middle-band-pass filter was applied, i.e., filtering signals with periods less than 30 days. Two main elements come out from this figure. In first place it verifies that for high activity level, 2001, the amplitude of the 27 day signal is significantly enhanced. This explains the 11 year modulation observed in Figure 3. In second place it shows that the amplitude of the 27 day signal is also modulated by a semiannual phenomenon: greater amplitudes during March and September.

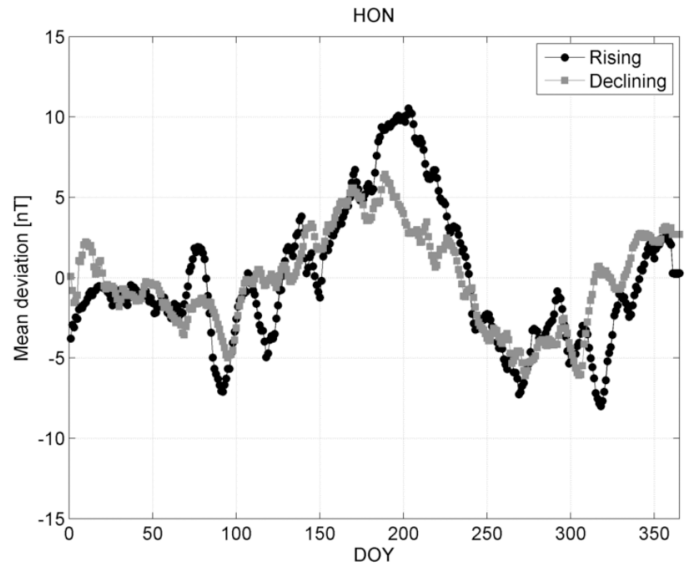

\subsection{Semiannual Anomaly Dependence on the Solar Magnetic Polarity}

[36] The next physical parameter evaluated was the polarity of the solar magnetic field. As it is known the polarity of the solar magnetic field flips its direction approximately every 11 years. The reversal moment is marked approximately by the start of the declining phase on the 11 year solar radiation cycle. The papers by Mursula et al. [2011] and Svaalgard [2011], are the most recent works dealing with some consequences of this problem.

[37] With the objective to analyze whether any polarity configuration favors the semiannual anomaly, the database for each fundamental observatory was binned according to the solar polarity with the following criteria (based on Oh and $Y i$ [2011]): periods with solar polarity parallel to the Earth's magnetic polarity: 1958-1969, 1981-1989, and 2001-2010; periods with solar polarity antiparallel to the Earth's magnetic

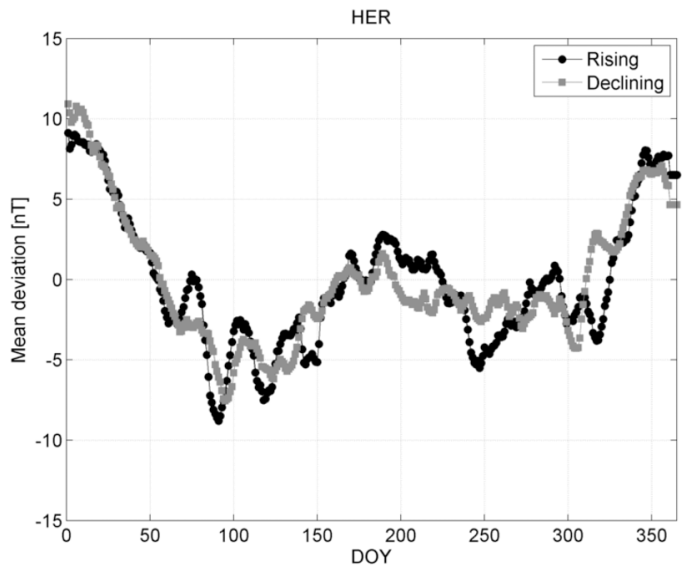

Figure 12. Mean deviations of the daily means of the H component for the HON observatory mapped against the DOY. The black dots correspond to declining phases of the solar radiation cycle, while the gray squares correspond to rising phases. For clarity a moving average with a 10 day window was applied to the data. 
polarity: 1948-1957, 1971-1981, and 1990-2000. Once again the $D s t>-50 \mathrm{nT}$ filter was applied. Then, the data processing scheme was applied to each data bin independently and the result is shown in Figure 11.

[38] From the comparison of the parallel and the antiparallel annual pattern it came out that there are not significant differences between them and that the general semiannual structure is clearly present on both curves. The most significant difference appears as a second-order effect associated to the 27 day signal.

\subsection{Semiannual Anomaly Dependence on the Phase (Rising or Declining) of the Solar Activity}

[39] The last dependence analyzed was on the rising/ declining phase of the solar radiation activity. For doing this the data set was binned according to (i) declining phase corresponding to the periods 1949-1954, 1958-1964, 1970 1975, 1982-1985, 1991-1995, and 2002-2007 and (ii) rising phase corresponding to the periods 1955-1957, 1966-1968, 1977-1980, 1987-1990, 1997-2000, and 2010. Then the usual data processing scheme was applied independently to each bin and the results are presented in Figure 12. The Dst $>-50 \mathrm{nT}$ filter was also applied.

[40] Once again it can be seen from this figure that although some differences exist between the declining and rising pattern they can be considered of a second order and that the general structure of the semiannual anomaly is clearly present for both phases. As it was found in the previous section the main differences appear associated to the 27 day (rotation period) signal.

\section{Summary and Conclusions}

[41] In section 2 we presented a new technique to reconstruct the annual pattern of the $\mathrm{H}$ component on the geomagnetic field and processed the historic data series of each of the four fundamental geomagnetic observatories. The dominant signal present is the semiannual anomaly (also known as semiannual variation). The comparison of the annual patterns led to the conclusion that the semiannual anomaly is present in the four data series. The unique Southern observatory (HER) presented significant differences, with an absolute maximum during December in opposition to the June absolute maximum of the others. In section 3 the analysis of a second Southern observatory, API, confirmed that HER annual pattern is characteristics of Southern observatories as already reported in Häkkinen et al. [2003]. As was mentioned in that section, this asymmetry has implications on the interpretation of the Dst index, because due to the three Northern observatories it might be biased toward Northern effects. Häkkinen et al. [2003] proposed a new Dst index based on 6 observatories (3 in each hemisphere) but we consider that this issue deserves a deeper analysis and would be the subject of a near-future paper.

[42] All through section 5, the annual patterns from HON and HER observatories were analyzed under different conditions that intended to cover the most important physical parameters characteristics of the Sun-Earth environment. Independently of periods with low and high geomagnetic activity (large geomagnetic storms), of low, moderate, and high solar activity (11 year solar cycle), of solar magnetic polarity parallel and antiparallel to the geomagnetic field (solar magnetic cycles) and of rising and declining phase of solar activity period, the semiannual anomaly in the $\mathrm{H}$ component is always present. This is the most important verification contained in this work.

[43] Also important is that its general structure, understanding by this the moments (DOY) of minima and maxima and the amplitude between minimums and maximums, is almost the same. Based on this, it can be concluded that the semiannual anomaly is dominated by the so-called nonstorm component and repeat itself year after year with an almost geometrical precision. Since there are several phenomena acting at the same time, the annual pattern observed during a particular year would appear slightly modified. This result gives evidences in favor of the equinoctial hypothesis.

[44] Another important conclusion is that the most affected phenomenon by the different physical conditions analyzed is the 27 day signal found in the patterns, which also presents a semiannual modulation.

[45] Acknowledgments. We acknowledge the archiving effort of the following observatories: Hermanus Magnetic Observatory (South Africa) for HER, U.S. Geological Survey (U.S.A.) for HON and SJG, Kakioka Magnetic Observatory (Japan) for KAK, and Institute of Geological Nuclear Sciences Ltd., Wellington (New Zealand), for API data series. We thank reviewer A. L. Clua de Gonzalez for her valuable comments and suggestions about our manuscript, which have enriched the original version. We also thank JGR Editor M. Fujimoto.

[46] Masaki Fujimoto thanks A. L. Clua de Gonzalez for assistance in evaluating this paper.

\section{References}

Bartels, J. (1932), Terrestrial-magnetic activity and its relations to solar phenomena, J. Geophys. Res., 37, 1-52, doi:10.1029/TE037i001p00001.

Chaman-Lal (2000), Sun-Earth geometry, geomagnetic activity, and planetary F2 layer ion density: Part I: Signatures of magnetic reconnection, J. Atmos. Sol. Terr. Phys., 62, 3-16.

Cliver, E. W., Y. Kamide, A. G. Ling, and N. Yokoyama (2001), Semiannual variation of the geomagnetic Dst index: Evidence for a dominant nonstorm component, J. Geophys. Res., 106(A10), 21,297-21,304, doi:10.1029/2000JA000358.

Cliver, E. W., Y. Kamide, and A. G. Ling (2002), The semiannual variation of geomagnetic activity: Phases and profiles for 130 years of $a a$ data, J. Atmos. Sol. Terr. Phys., 64, 47-53, doi:10.1016/S1364-6826(01) 00093-1.

Cliver, E. W., L. Svalgaard, and A. G. Ling (2004), Origins of the semiannual variation of geomagnetic activity in 1954 and 1996, Ann. Geophys., 22, 93-100, doi:10.5194/angeo-22-93-2004.

Clúa de Gonzalez, A. L., V. M. Silbergleit, W. D. Gonzalez, and B. T. Tsurutani (2001), Annual variation of geomagnetic activity, J. Atmos. Sol. Terr. Phys., 63, 367-374, doi:10.1016/S1364-6826(00)00190-5.

Cortie, A. L. (1912), Sun-spots and terrestrial magnetic phenomena, 1898-1911: The cause of the annual variation in magnetic disturbances, Mon. Not. R. Astron. Soc., 73, 52-60.

Gonzalez, W. D., J. A. Joselyn, Y. Kamide, H. W. Kroehl, G. Rostoker, B. T. Tsurutani, and V. M. Vasyliunas (1994), What is a geomagnetic storm?, J. Geophys. Res., 99(A4), 5771-5792, doi:10.1029/93JA02867.

Häkkinen, L. V. T., T. I. Pulkkinen, R. J. Pirjola, H. Nevanlinna, E. I. Tanskanen, and N. E. Turner (2003), Seasonal and diurnal variation of geomagnetic activity: Revised Dst versus external drivers, J. Geophys. Res., 108(A2), 1060, doi:10.1029/2002JA009428.

Lal, C. (1998), Solar wind and equinoctial maxima in geophysical phenomena, J. Atmos. Sol. Terr. Phys., 60, 1017-1024, doi:10.1016/S1364-6826(98) 00046-7.

Lyatsky, W., and A. Tan (2003), Latitudinal effect in semiannual variation of geomagnetic activity, J. Geophys. Res., 108(A5), 1204, doi:10.1029/ 2002JA009467.

Mayaud, P. N. (1980), Derivation, Meaning, and Use of Geomagnetic Indices, Geophys. Monogr. Ser., vol. 22, 154 pp., AGU, Washington, D. C., doi:10.1029/GM022.

Mursula, K., L. Holappa, and A. Karinen (2008), Correct normalization of the Dst index, Astrophys. Space Sci. Trans., 4, 41-45, doi:10.5194/ astra-4-41-2008. 
Mursula, K., E. Tanskanen, and J. J. Love (2011), Spring-fall asymmetry of substorm strength, geomagnetic activity and solar wind: Implications for semiannual variation and solar hemispheric asymmetry, Geophys. Res. Lett., 38, L06104, doi:10.1029/2011GL046751.

O'Brien, T. P., and R. L. McPherron (2002), Seasonal and diurnal variation of Dst dynamics, J. Geophys. Res., 107(A11), 1341, doi:10.1029/ 2002JA009435.

Oh, S. Y., and Y. Yi (2011), Solar magnetic polarity dependency of geomagnetic storm seasonal occurrence, J. Geophys. Res., 116, A06101, doi:10.1029/2010JA016362.

Russell, C. T. (1989), The universal time variation of geomagnetic activity, Geophys. Res. Lett., 16(6), 555-558, doi:10.1029/GL016i006p00555.

Russell, C. T., and R. L. McPherron (1973), Semiannual variation of geomagnetic activity, J. Geophys. Res., 78, 92-108, doi:10.1029/ JA078i001p00092.
Sabine, E. (1856), On periodical laws discoverable in the mean effects of the larger magnetic disturbances. No. III, Philos. Trans. R. Soc. London B, 146, 357-374, doi:10.1098/rstl.1856.0016.

Sugiura, M., and T. Kamei (1991), Equatorial Dst Index: 1957-1986, Int Assoc. Geomagn. Aeron. Bull., vol. 40, edited by A. Berthelier and M. Menvielle, 245 pp., Int. Union of Geod. and Geophys. Publ. Off., Saint-Maur-des-Fossés, France.

Svalgaard, L. (1977), Geomagnetic activity: Dependence on solar wind parameters, in Coronal Holes and High Speed Wind Streams, edited by J. B. Zirker, pp. 371-441, Colo. Assoc. Univ. Press, Boulder.

Svalgaard, L. (2011), Geomagnetic semiannual variation is not overestimated and is not an artifact of systematic solar hemispheric asymmetry, Geophys. Res. Lett., 38, L16107, doi:10.1029/2011GL048616.

Yoshida, A. (2009), Physical meaning of the equinoctial effect for semi-annual variation in geomagnetic activity, Ann. Geophys., 27, 1909-1914, doi:10.5194/angeo-27-1909-2009. 